\title{
Efficacy of artemether-lumefantrine as a treatment for uncomplicated Plasmodium vivax malaria in eastern Sudan
}

Tajeldin M Abdallah ${ }^{1}$, Abdel Aziem A Ali ${ }^{1}$, Mohammed Bakri ${ }^{1}$, Gasim I Gasim ${ }^{1,3}$, Imad R Musa ${ }^{1,4}$ and Ishag Adam²

\begin{abstract}
Background: Artemisinin-based combination therapy (ACT) is the treatment of choice for uncomplicated Plasmodium falciparum malaria in most areas of the world, where malaria is endemic, including Sudan. However, few published data are available on the use of ACT for treatment of $P$. vivax malaria.

Methods: This study was conducted at a health centre in Kassala, eastern Sudan, from October to December 2011. Patients with uncomplicated $P$. vivax malaria received artemether-lumefantrine (AL) tablets (containing $20 \mathrm{mg}$ artemether and $120 \mathrm{mg}$ lumefantrine) and were monitored for 28 days.

Results: Out of the 43 cases enrolled in this study, 38 completed the 28-day follow-up. Their mean age was 25.1 years (SD: 1.5). On day 3 following AL treatment, all of the patients were afebrile and aparasitaemic. By day 28, all 38 patients exhibited adequate clinical and parasitological responses to AL treatment. The cure rate was 100\% and $88.4 \%$ for the per protocol analysis andfor the intention to treat analysis, respectively. Mild adverse effects (nausea, vomiting, abdominal pain, dizziness and/or rash) that resolved spontaneously were observed in four (10.5\%) of the patients.
\end{abstract}

Conclusion: AL combination therapy was fully effective for treatment of $P$. vivax malaria in the study in eastern Sudan.

Trial registration: Trial. Gov: NCT01625871

Keywords: Efficacy, Artemether-lumefantrine, Malaria, P. vivax, Sudan.

\section{Background}

Plasmodium vivax infection is a major global health problem. This species of parasite has the broadest geographic distribution of the five malaria species known to infect humans [1]. There are about 2.5 billion people at risk of malaria and an estimated 80 to 300 million clinical cases of $P$. vivax annually [2,3]. Although $P$. vivax is mainly endemic in Southeast Asia and Latin America [3], it has recently been observed in Ethiopia and Sudan [4-8].

Malaria is a important health problem in Sudan, and in 2002, an estimated 9 million disease episodes and 44,000 deaths from the disease occurred [9]. The spread of multidrug-resistant Plasmodium falciparum malaria in Sudan $[10,11]$ has led to adoption of artemisinin-based

\footnotetext{
* Correspondence: ishagadam@hotmail.com

${ }^{2}$ Faculty of Medicine, University of Khartoum, P.O. Box 102, Khartoum, Sudan Full list of author information is available at the end of the article
}

combination therapy (ACT), with artesunate-sulphadoxine-pyrimethamine (AS-SP) and artemether-lumefantrine (AL) becoming the recommended first- and secondline treatments for uncomplicated $P$. falciparum malaria, respectively.

Early diagnosis and effective treatment with an appropriate drug is one of the main components of the World Health Organization's strategy to reduce malaria related mortality [12]. Episodes of $P$. vivax infection should prompt urgent treatment with effective anti-malarial medication [13]. While most malaria endemic countries have adopted ACT to reduce the risk of multidrug resistant strains of P. falciparum occurring [10], chloroquine remains the first-line treatment for $P$. vivax in most endemic countries. However, there is growing evidence that the efficacy of chloroquine against $P$. vivax is declining in many areas, especially Southeast Asia [14-16]. 
Hence, the use of ACT against both $P$. vivax and $P$. falciparum is preferred, especially in a country like Sudan where chloroquine is no longer registered or available [17]. The far-reaching adoption of ACT as an effective first-line therapy for P. falciparum has led to a closer examination of their role in the management of $P$. vivax malaria. AL is one such combination therapy that is widely used to treat uncomplicated $P$. falciparum malaria, and is the first-line treatment for $P$. vivax malaria according to the national malaria control programme in Sudan. However, there is no published data on the efficacy of $\mathrm{AL}$ for the treatment of $P$. vivax malaria in Sudan. Therefore, the efficacy of AL for the treatment of uncomplicated $P$. vivax malaria was investigated in Sudan. The study was conducted at a health centre in eastern Sudan, an area characterized by unstable malaria transmission [18]. Recently, however, the most severe form of $P$. vivax malarial disease has been observed in the area [8]. The study is of paramount importance because it aims to provide Sudanese health care professionals and public health planners with the fundamental data necessary for developing an effective programme for malaria control.

\section{Methods}

This observational (without sample size calculation) clinical trial was conducted at the Fatima Eldeaig Health Centre in Kassala, eastern Sudan (located $600 \mathrm{~km}$ from Khartoum, Sudan) and took place from October to December 2011 (Figure 1). The World Health Organization (WHO) guidelines for assessment of the efficacy of anti-malarial drugs were adopted [19]. Febrile patients (axillary temperature $\geq 37.5^{\circ} \mathrm{C}$ ) with confirmed blood films for $P$. vivax mono-infection and willing to participate in the study were enrolled. Those individuals with severe malnutrition, pregnancy, or with a history of allergy and/or intolerance to the drugs were excluded. Informed consent was obtained from all of the patients, or in the case of children, their guardians. Structured questionnaires were used together

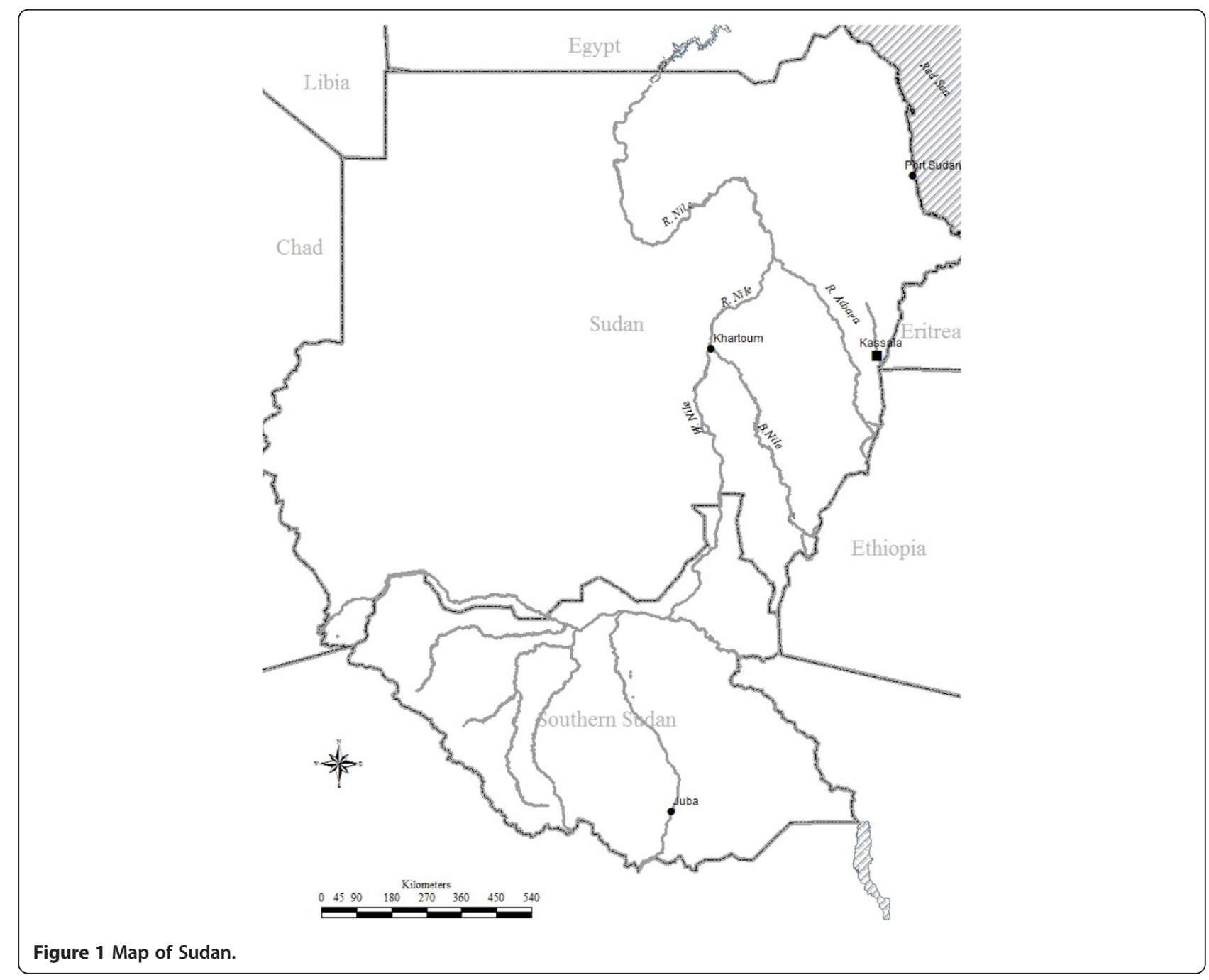


with socio-demographic characteristics, medical histories (including the duration of the illness) and physical findings.

Thick and thin blood smears were prepared and stained with $2 \%$ Giemsa solution for 30 minutes. Thick blood films were used for counting parasite numbers and for estimating parasite densities. Gametocytes were counted against 200 leukocytes, and parasite densities estimated using 8,000 white blood cells (WBCs)/ $\mu \mathrm{L}$ of blood as the multiplier. Smears were considered negatives if no parasites could be seen in 100 high-power microscopic fields. Blood films were double checked (blind) by two independent examiners, and discordant results were agreed on after evaluating the evidence of each examiner. Parasite densities differing by more than $10 \%$ between the different microscopists were evaluated by a third microscopist, whose result was deemed to be final.

\section{Treatment}

AL (Coartem ${ }^{\circledR}$; Novartis, Basel, Switzerland) was administered over a three-day period. AL tablets contained $20 \mathrm{mg}$ of artemether and $120 \mathrm{mg}$ of lumefantrine. Patients were weighed and those corresponding to 5-14, 15-24, 25-34 or $>35 \mathrm{~kg}$ were each given six doses of AL (at $0,8,24,36,48$ and $60 \mathrm{~h}$ ), each dose consisted of one, two, three or four tablets, respectively. The morning dose was given at the health Centre under the direct supervision of a medical officer. Young children who could not swallow tablets were given suspensions created by crushing and dissolving their tablets in water. All patients were observed for vomiting for $1 \mathrm{~h}$ after each treatment. If vomiting occurred within $30 \mathrm{~min}$ of a dose, a second full dose was administered. If vomiting occurred 31-60 min after a dose, however, only half a dose was given. If vomiting occurred after a repeated (full or half) dose, the patient was given a quinine infusion and excluded from the study. These instructions were given to all of the patients (or guardian in the case of children) because the evening dose was given unsupervised. Patients also received unsupervised primaquine tablets $(15 \mathrm{mg})$ daily for 14 days.

\section{Treatment outcomes}

Patients were asked to return on days 1, 2, 3, 7, 14, 21 and 28 of the study, or at any other time if they felt unwell. During each visit, the participants' temperatures were measured and blood smears were prepared and checked as described above. Treatment outcomes were classified according to WHO guidelines for evaluation of the therapeutic efficacy of antimalarial drugs in the treatment of uncomplicated malaria [19]. During followup, the patients were asked if they had any adverse effects that might have been expected from the treatment itself (nausea, vomiting, abdominal pain, dizziness and rash). These effects were considered to be AL-related if they had not been reported when the patient first presented for treatment at the Health Centre. The primary outcome was the cure rate, which was defined as the percentage of the patients completing follow-up that had adequate clinical and parasitological responses (ACPR) after 28 days. Secondary outcomes comprised the frequencies of early treatment failure, late clinical response, late parasitological response, and adverse events, and the time taken to reduce fever and clear parasitaemia. Early treatment failure was defined as the development of danger signs or severe malaria, in the presence of parasitaemia, on days 1, 2 and/or 3, a day- 2 parasitaemia that was higher than the day- 0 , the presence of both fever and parasitaemia on day 3 , and/or a day-3 parasitaemia that was $20 \%$ of the day- 0 value. A late treatment failure was defined as the development of danger signs or severe malaria, in the presence of parasitaemia, after day 3 , the presence of both fever and parasitaemia on or after day 4 , and/or the presence of parasitaemia after day 7 .

\section{Data analysis}

Data were entered into a computer database and analysed using SPSS software (version 20, Chicago, IL, USA). Mean and SD were calculated for the variables presented. The intention-to-treat analysis included all enrolled patients who met the inclusion criteria and took at least one full dose of AL. Patients lost to follow-up or withdrawn from the study were considered to be treatment failures. The per protocol (PP) analysis of outcomes included data for patients who had completed the follow-up. Those patients lost to the follow-up or were withdrawn because of protocol violations were excluded from the PP analysis.

\section{Ethics}

The current study received ethical approval from the Health Research Board at the Ministry of Health in Kassala state, eastern Sudan. The trial was registered at Trial. Gov: NCT01625871.

\section{Results}

During the study period, 1,185 febrile patients presented to the health centre, of which 251 had uncomplicated diagnoses of malaria confirmed. Out of these 251 patients, 204, 43, and four were uncomplicated P. falciparum, uncomplicated $P$. vivax and mixed $P$. falciparum and $P$. vivax infections, respectively (Figure 2 ). Therefore, the $P$. falciparum: $P$. vivax ratio was $4.7: 1$. Of the 43 enrolled patients with uncomplicated $P$. vivax malaria, 38 (88.37\%) completed the 28 days follow-up, but the remaining 5 were lost to follow- up. The base line characteristics of the study participants are shown in Table 1 . The range of the age, weight and parasitaemia was 4-60 years, $13-95 \mathrm{~kg}$ years, and $200-8,480.0$ rings/ $\mu \mathrm{l}$, respectively. The majority 


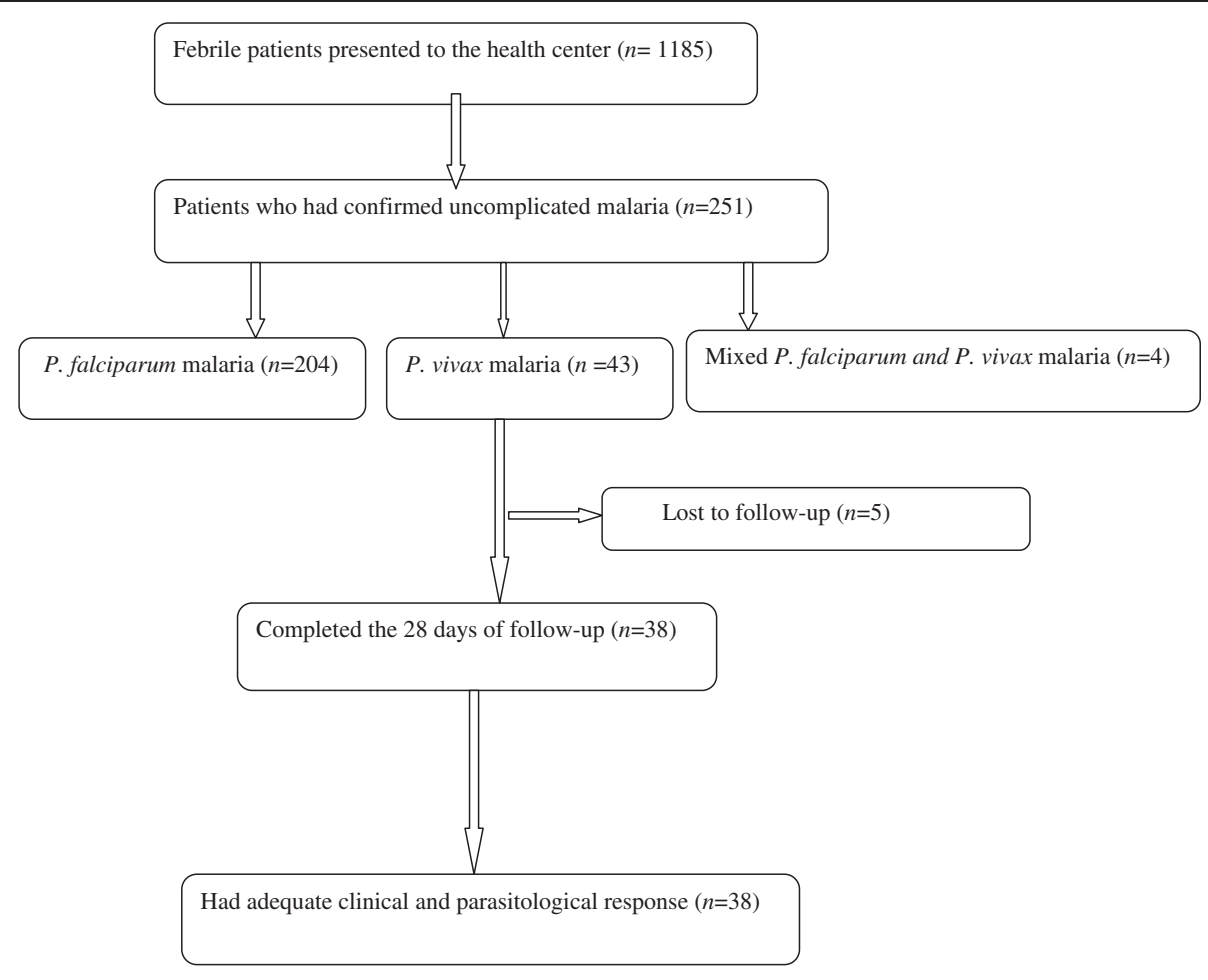

Figure 2 Patient enrollment and follow-up during the study.

$(24,63.2 \%)$ of these patients were male. Only one patient was aged less than five years (a 4-year old). Excluding those with gametocytes on day 0 (two patients), no new carriers were identified during the 28-day follow-up. All of the day 0 slides that were submitted to the National Laboratory at the National Malaria Control Programme for quality control were identified as containing the same species of malaria parasite (i.e., $P$. vivax).

On day 1 all patients were afebrile, but 2 (5.3\%) of them were parasitaemic. By day 3, all patients were afebrile and aparasitaemic. All of the patients 38 (100\%) responded to treatment with $\mathrm{AL}$ and had ACPR. The cure rate was $100 \%$ and $88.4 \%$ for the per protocol analysis and for the intention to treat analysis, respectively. Mild adverse effects (nausea, vomiting, abdominal pain, dizziness and/or rash) that resolved spontaneously were observed in four (10.5\%) patients.

Table 1 Baseline characteristics of patients treated with artemether-lumefantrine in eastern Sudan

\begin{tabular}{ll}
\hline Variable & Mean (SD) \\
\hline Age in years & $25.1(1.5)$ \\
Weight in $\mathrm{kg}$ & $50.2(2.5)$ \\
Temperature ${ }^{\circ} \mathrm{C}$ & $38.5(0.6)$ \\
Duration of the illness in days prior to diagnosis & $4.1(2.4)$ \\
Geometric mean of asexual-stage parasitaemia per $\mu \mathrm{L}$ & $3272.0(2029.3)$ \\
\hline
\end{tabular}

\section{Discussion}

The current study showed full efficacy (per protocol analyses) of the AL combination for treatment of $P$. vivax in eastern Sudan. Consistent with a recent study, the present study showed that $P$. vivax is a health problem and that its ratio to $P$. falciparum has increased [8]. Previous studies showed that AL had a high efficacy (98.7\%) for treatment of uncomplicated P. falciparum malaria in eastern Sudan as well as other regions of the country [20,21]. Interestingly, in neighbouring Ethiopia, a higher failure rate was observed (19\%) when AL was compared with chloroquine for treatment of uncomplicated $P$. vivax malaria [4]. The high AL failure rate in Ethiopia was attributed to the need for patients to take the evening dose of the drug unsupervised [4]. In this context, it is noteworthy that the emergence of chloroquineresistant $P$. vivax was first confirmed in Ethiopia [22]. The full efficacy of AL for the treatment of $P$. vivax malaria in the present study is consistent with other studies that showed a rapid clearance of $P$. vivax parasitaemia and fever and an overall high cure rate for AL treatment of P. vivax malaria $[13,23]$.

Chloroquine remains the drug of choice for treating $P$. vivax infection according to $\mathrm{WHO}$ guidelines and it is still effective in most malaria endemic areas [24,25]. Unfortunately, substantial levels of chloroquine-resistant P. vivax parasites have been observed in South East Asia [26-28]. In addition, some studies have reported 
chloroquine treatment failure for $P$. vivax malaria in Africa $[4,22,29]$. Therefore, the idea of using a single first-line therapy that is effective against both $P$. vivax and $P$. falciparum is attractive, especially in settings where two malaria parasite species are co-endemic. ACT is highly effective against uncomplicated $P$. falciparum malaria and, as a consequence of high levels of chloroquine resistance, is now widely adopted as the first-line therapy in most malaria endemic countries, including Sudan [10]. Indeed, some countries such as Papua New Guinea and Indonesia have adopted ACT as the first-line therapy for P. vivax and P. falciparum malaria [16]. A policy whereby use of a unified first-line therapy based on ACT was implemented could have great public health value in that it would simplify treatment, management and logistics of malaria disease control. It should be mentioned that chloroquine is no longer registered or available for use in Sudan, so as to avoid the problem of $P$. falciparum infection being treated with chloroquine.

The limitations of this study concern its nonrandomized character and small sample size; however, with a lack of published data about the efficacy of AL against $P$. vivax malaria, this study provides useful insight into AL treatment of $P$. vivax infection. Secondly, the 28-day follow-up of patients treated with $\mathrm{AL}$ is another limitation; a longer follow-up period could be advantageous as it would provide more information about AL treatment outcomes. Thirdly, in this study, patients were treated with primaquine without prior assessment of their G6PD enzyme levels, because G6PD enzyme tests were not available.

\section{Conclusion}

Although this study did not detect any treatment failures during treatment of uncomplicated $P$. vivax malaria using AL in this area of Sudan.

\section{Competing interest}

The authors declare that they have no competing interests.

\section{Authors' contributions}

$\mathrm{TM}, \mathrm{GIG}$, IA designed the study, AAA, IRM and MB conducted the clinical work. All of the authors drafted and approved the manuscript.

\section{Author details}

${ }^{1}$ Faculty of Medicine, Kassala University, Kassala, Sudan. ${ }^{2}$ Faculty of Medicine, University of Khartoum, P.O. Box 102, Khartoum, Sudan. ${ }^{3}$ Faculty of Medicine, Qassim University, Qassim, Qassim, Kingdom of Saudi Arabia. ${ }^{4}$ Buraidah Central Hospital, Buraidah, Kingdom of Saudi Arabia.

Received: 20 September 2012 Accepted: 3 December 2012 Published: 5 December 2012

\section{References}

1. Guerra CA, Howes RE, Patil AP, Gething PW, Van Boeckel TP, Temperley WH, Kabaria CW, Tatem AJ, Manh BH, Elyazar IR, Baird JK, Snow RW, Hay SI: The international limits and population at risk of Plasmodium vivax transmission in 2009. PLoS Negl Trop Dis 2010, 4:e774.

2. Mendis K, Sina BJ, Marchesini P, Carter R: The neglected burden of Plasmodium vivax malaria. Am J Trop Med Hyg 2001, 64:97-106.
3. Mueller I, Galinski MR, Baird JK, Carlton JM, Kochar DK, Alonso PL, del Portillo HA: Key gaps in the knowledge of Plasmodium vivax, a neglected human malaria parasite. Lancet Infect Dis 2009, 9:555-566.

4. Yohannes AM, Teklehaimanot A, Bergqvist Y, Ringwald P: Confirmed vivax resistance to chloroquine and effectiveness of artemether-lumefantrine for the treatment of vivax malaria in Ethiopia. Am J Trop Med Hyg 2011, 84:137-140.

5. Nigatu W, Abebe M, Dejene A: Plasmodium vivax and P. falciparum epidemiology in Gambella, south-west Ethiopia. Trop Med Parasitol 1992, 43:181-185.

6. Jima D, Getachew A, Bilak H, Steketee RW, Emerson PM, Graves PM, Gebre T, Reithinger R, Hwang J: Ethiopia Malaria Indicator Survey Working Group: Malaria indicator survey 2007 Ethiopia: coverage and use of major malaria prevention and control interventions. Malar J 2010, 9:58.

7. Animut A, Mekonnen Y, Shimelis D, Ephraim E: Febrile illnesses of different etiology among outpatients in four health centers in Northwestern Ethiopia. J Infect Dis 2009, 62:107-110.

8. Mahgoub H, Gasim GI, Musa IR, Adam I: Severe Plasmodium vivax malaria among Sudanese children at New Halfa Hospital Eastern Sudan. Parasit Vectors 2012, 5:154.

9. Abdalla SI, Malik EM, Ali KM: The burden of malaria in Sudan: incidence, mortality and disability-adjusted life-years. Malar J 2007, 6:97.

10. Adam I, Salah MT, Eltahir HG, Elhassan AH, Elmardi KA, Malik EM: Dihydroartemisinin-piperaquine versus artemether-lumefantrine, in the treatment of uncomplicated Plasmodium falciparum malaria in central Sudan. Ann Trop Med Parasitol 2010, 104:319-326.

11. Adam I, Osman ME, Elghazali G, Ahmed Gl, Gustafson LL, Elbashir Ml: Efficacies of chloroquine, sulphadoxine-pyrimethamine and quinine for the treatment of uncomplicated Plasmodium falciparum malaria in eastern Sudan. Ann Trop Med Parasitol 2004, 98:661-666.

12. World Health Organization: Implementation of the Global Malaria Control Strategy. Report of a WHO Study Group on the Implementation of the Global Plan of Action for Malaria Control 1993-2000. Technical Report Series no. 839. Geneva: World Health Organization; 1993.

13. Quique B: The use of artemether-lumefantrine for the treatment of uncomplicated Plasmodium vivax malaria. PLoS Negl Trop Dis 2011, 12:e1325.

14. Rieckmann $\mathrm{KH}$, Davis DR, Hutton DC: Plasmodium vivax resistance to chloroquine? Lancet 1989, 2:1183-1184.

15. Price RN, Douglas NM, Anstey NM: New developments in Plasmodium vivax malaria: severe disease and the rise of chloroquine resistance. Curr Opin Infect Dis 2009, 22:430-435.

16. Douglas NM, Anstey NM, Angus BJ, Nosten F, Price RN: Artemisinin combination therapy for vivax malaria. Lancet Infect Dis 2010, 10:405-416.

17. Malik EM, Mohamed TA, Elmardi KA, Mowien RM, Elhassan AH, Elamin SB, Mannan AA, Ahmed ES: From chloroquine to artemisinin-based combination therapy: the Sudanese experience. Malar J 2006, 5:6.

18. Osman MM, Nour BY, Sedig MF, De Bes L, Babikir AM, Mohamedani AA, Mens PF: Informed decision-making before changing to RDT: a comparison of microscopy, rapid diagnostic test and molecular techniques for the diagnosis and identification of malaria parasites in Kassala, eastern Sudan. Trop Med Int Health 2010, 15:1442-1448.

19. WHO: Methods for surveillance of antimalarial drug efficacy. WHO.Int/ Publications/; 2009. http://www.who.int/malaria/publications/atoz/ 9789241597531/en/index.html.

20. Sagara I, Rulisa S, Mbacham W, Adam I, Sissoko K, Maiga H, Traore OB, Dara N, Dicko YT, Dicko A, Djimdé A, Jansen FH, Doumbo OK: Efficacy and safety of a fixed dose artesunate-sulphamethoxypyrazine-pyrimethamine compared to artemether-lumefantrine for the treatment of uncomplicated falciparum malaria across Africa: a randomized multicentre trial. Malar J 2009, 8:63.

21. Elamin SB, Malik EM, Abdelgadir T, Khamiss AH, Mohammed MM, Ahmed ES, Adam I: Artesunate plus sulfadoxine-pyrimethamine for treatment of uncomplicated Plasmodium falciparum malaria in Sudan. Malar J 2005, 4:41.

22. Teka H, Petros B, Yamuah L, Tesfaye G, Elhassan I, Muchohi S, Kokwaro G, Aseffa A, Engers $\mathrm{H}$ : Chloroquine-resistant Plasmodium vivax malaria in Debre Zeit, Ethiopia. Malar J 2008, 7:220.

23. Krudsood S, Tangpukdee N, Muangnoicharoen S, Thanachartwet V, Luplertlop N, Srivilairit S, Wilairatana P, Kano S, Ringwald P, Looareesuwan S: Clinical efficacy of chloroquine versus artemether-lumefantrine for Plasmodium vivax treatment in Thailand. Korean J Parasitol 2007, 45:111-114. 
24. Alvarez G, Piñeros JG, Tobón A, Ríos A, Maestre A, Blair S, Carmona-Fonseca $\mathrm{J}$ : Efficacy of three chloroquine-primaquine regimens for treatment of Plasmodium vivax malaria in Colombia. Am J Trop Med 2006, 76:605-609.

25. Naing C, Aung K, Win DK, Wah MJ: Efficacy and safety of chloroquine for treatment in patients with uncomplicated Plasmodium vivax infections in endemic countries. Trans R Soc Trop Med 2010, 104:695-705.

26. Baird JK: Chloroquine resistance in Plasmodium vivax. Antimicrob Agents Chemother 2004, 48:4075-4083.

27. Baird JK: Resistance to therapies for infection by Plasmodium vivax. Clin Microbiol Rev 2009, 22:508-534.

28. Sutanto I, Endawati D, Ling LH, Laihad F, Setiabudy R, Baird JK: Evaluation of chloroquine therapy for vivax and falciparum malaria in southern Sumatra, western Indonesia. Malar J 2010, 9:52.

29. Ketema T, Bacha K, Birhanu T, Petros B: Chloroquine-resistant Plasmodium vivax malaria in Serbo town, Jimma zone, south-west Ethiopia. Malar J 2009, 8:177.

doi:10.1186/1475-2875-11-404

Cite this article as: Abdallah et al:: Efficacy of artemether-lumefantrine as a treatment for uncomplicated Plasmodium vivax malaria in eastern Sudan. Malaria Journal 2012 11:404.

\section{Submit your next manuscript to BioMed Central and take full advantage of:}

- Convenient online submission

- Thorough peer review

- No space constraints or color figure charges

- Immediate publication on acceptance

- Inclusion in PubMed, CAS, Scopus and Google Scholar

- Research which is freely available for redistribution 